\title{
Repercusión en la calidad de vida, salud y práctica de actividad física del confinamiento por Covid-19 en España Effects on quality of life, health and practice of physical activity of Covid-19 confinement in Spain \\ *Marta García-Tascón, **Cristina Mendaña-Cuervo, **César Sahelices-Pinto, ***Ana-M Magaz-González \\ "Universidad Pablo de Olavide (España), ${ }^{* *}$ Universidad de León (España), ${ }^{* * *}$ Universidad de Valladolid (España)
}

Resumen: En España, la declaración del estado de alarma por la pandemia Covid-19, supuso que entre el 14 de marzo y el 2 de mayo de 2020, 47 millones de habitantes estuvieran confinados y sólo pudieran salir a cubrir necesidades básicas. Ante este hecho es oportuno analizar su impacto sobre la salud, tomando en consideración cómo afectó a la práctica de la actividad física. Se administró un cuestionario ad hoc. Los participantes fueron 1046 personas (hombre 48.57\% y mujer 51.43\%) y con una edad media de 40 ( \pm DT 13.35 años). Respecto a la práctica de actividad física, destaca una significativa disminución de intensidad y cantidad ( $\mathrm{p}<.001$ ), y una modificación del tipo de actividad física realizada, pasando de deportes cardiovasculares y de aptitud muscular a prácticas de gimnasias suaves y flexibilidad. Aumentó el porcentaje total de personas que no realizaron nada de actividad física. Aun disponiendo de más tiempo libre, las personas en situación de ERTE, disminuyeron significativamente su cantidad de actividad física realizada $(\mathrm{p}<.001)$. Estos resultados permiten concluir que el confinamiento supuso una modificación de hábitos de práctica de actividad física, una disminución de los valores recomendados como saludables, lo que conllevó implicaciones sobre la salud y la calidad de vida. Se ofrece información que ayudará a entender estos nuevos comportamientos y que facilitará a los gestores estrategias innovadoras para activar la vuelta a niveles anteriores de actividad física, su incremento y, en definitiva, la reactivación del sector deportivo.

Palabras clave: Covid-19; estado alarma; práctica física, ejercicio, bienestar.

\begin{abstract}
In Spain, the declaration of the state of alarm due to the Covid-19 pandemic, meant that between $14^{\text {th }}$ of March and $2^{\text {nd }}$ of May 2020, 47 million inhabitants were confined at home and could only go out to cover basic needs. This unprecedented event has led to a paradigm shift, and it is appropriate to analyze its impact on health, considering how it has affected the practice of physical activity. An ad hoc questionnaire was administered. The participants were 1046 people (48.57\% male and 51.43\% female) and the average age of 40 ( \pm DT 13.35 years). Regarding the practice of physical activity, a significant decrease in intensity and quantity stands out $(\mathrm{p}<.001)$, and a modification of the type of physical activity performed, going from cardiovascular sports and muscular fitness to soft gymnastics and flexibility practices. The total percentage of people who did no physical activity at all, increased. In addition, although more free time was available, people in ERTE situation significantly decreased their amount of physical activity performed $(\mathrm{p}<.001)$. These results allow us to conclude that confinement has meant a modification of physical activity practice habits and a decrease in the recommended values of healthy healthy, and therefore has had implications on health and quality of life. This information will help to understand these new behaviors in order for managers to implement innovative strategies to reactivate the return to previous levels of physical activity, its increase and, in short, the reactivation of the sports sector.
\end{abstract}

Keywords: Covid-19, state of alarm, physical practice, exercise, well-being.

\section{Introducción}

La declaración por la Organización Mundial de la Salud (OMS) de pandemia internacional de la enfermedad por la Covid-19 (OMS, 2020) provocó la mayor reclusión de la población a nivel mundial en diferentes grados según gobiernos. En España, se declaró el primer estado de alarma para proteger la salud y la seguridad de los ciudadanos, a través del Real Decreto [RD]

Fecha recepción: 01-03-21. Fecha de aceptación: 11-05-21

Marta García-Tascón

margata@upo.es
463/2020, de 14 de marzo (Ministerio de la Presidencia, Relaciones con la Cortes y Memoria Democrática [MP], 2020a) y con ello el confinamiento de toda la población española. Esta medida anunciada para 15 días, fue prorrogada mediante sucesivos decretos hasta el día 21 de junio de 2020 en que se puso fin al primer estado de alarma (MP, 2020b). De esta manera, durante un mes y medio sólo se pudo salir a la calle para cubrir las necesidades básicas señaladas en el art. 7 del RD 463/ 2020 (MP, 2020a), restringiendo la libertad de movimiento al hogar. Posteriormente, se mantuvieron limitaciones en distinto grado hasta junio. Así, la Orden SND/380/2020, de 30 de abril (Ministerio de Sanidad, 
2020a) permitió, desde el 2 de mayo de 2020, realizar actividad física (AF) no profesional al aire libre con restricciones horarias, de forma individual y sin contacto.

La población española se tuvo que adaptar a este escenario insólito y extraordinario. Las restricciones en la vida diaria fueron de gran alcance (cierre de ciudades, escuelas, comercios, infraestructuras no esenciales como gimnasios, parques o restaurantes) transformando las relaciones laborales, formas de trabajo y limitando el contacto social y personal (OECD, 2020). También se alteraron patrones de alimentación, de práctica de $\mathrm{AF}$ y sueño, impactando directamente sobre la salud, física y emocional de las personas (Frontiers, 2020).

La estructura productiva se adaptó bruscamente al confinamiento, alterando los procesos de producción y venta, afectando al empleo y a la economía en casi todos los sectores. La primera estimación de la variación del 2020 es de $-10.8 \%$ respecto al año anterior (Instituto Nacional de Estadística [INE], 2021a) y el sector fitness tasa en $-44 \%$ la facturación para los siguientes doce meses, asumiendo pérdidas de 1.108 millones de euros (Muñiz, 2020). El parón productivo impuesto desencadenó un aumento de los expedientes de regulación de empleo temporal (ERTE), rozando los 4 millones en abril de 2020 (Martín, 2020). El 98\% de gimnasios y centros deportivos hacen uso de esta medida y entre el $75-100 \%$ de su plantilla se acoge a ella (JiménezGutiérrez et al., 2020).

El sector de la actividad físico-deportiva (AFD) quedó dañado en todas sus manifestaciones, desde el ámbito educativo (Alarcón-Meza \& Hall-López, 2021; ArrasVota et al., 2021; Baena-Morales et al., 2021; Hortigüela-Alcalá et al., 2021) hasta los juegos olímpicos de Tokio que se aplazaron (Bosch et al., 2020; Consejo General de la Educación Física y Deportiva [COLEF], 2020; International Olympic Committee [IOC], 2020; Jiménez-Gutiérrez et al., 2020).

La industria deportiva no pudo generar el servicio en su formato tradicional (estadios, gimnasios y piscinas cerrados y espacios al aire libre precintados) y consumidores y productores tuvieron que adaptarse al estado de alarma sin previo aviso (García-Tascón, 2020; López, 2020a; Moratalla \& Íbero, 2020). Por una parte, los practicantes tuvieron que variar sus hábitos de consumo deportivo: modalidades, horas, intensidad, escenarios (Astiazarán \& Ara, 2020). Por ejemplo, en España, durante las semanas de confinamiento varió la tipología de AF realizada (Instituto de Biomecánica deValencia [IBV], 2020; Magaz-González \& García-Tascón, 2020), bajaron la intensidad y el número de horas semanales de práctica (Castañeda-Babarro et al., 2020; López-Bueno et al., 2020; Magaz-González \& García-Tascón, 2020; Pérez-Rodrigo et al., 2021) y se dieron un $38 \%$ menos de pasos por semana que antes de la reclusión (Fitbit Staff, 2020). Por otra parte, gracias a las tecnologías de la información y las comunicaciones (TICs), algunas organizaciones lanzaron su oferta en entornos web, con lo que ciertas actividades habituales se pudieron mantener: entrenamiento, readaptación funcional, salud, recreación, etc.

Ahora bien, aunque muchas organizaciones consiguieron la inmersión digital en tiempo récord, la gran mayoría no disponía de una estrategia tecnológica (Fagde, 2020a; Jiménez-Barreto \& Borges, 2021; López, 2020b; Moreno, 2020). Además, la percepción que los usuarios tienen de la accesibilidad, la utilidad y la facilidad del uso de la tecnología para cubrir sus necesidades de AF (Valcarce, 2020), el comportamiento social adherido a la práctica (donde la relación social es una de las principales motivaciones para la misma) y el comportamiento tradicional de los practicantes que no incluye el consumo de AFD virtual desde sus hogares (Astiazarán \& Ara, 2020; Magaz-González et al., 2020) supuso que, a pesar de oferta e iniciativas virtuales privadas y públicas (Consejo-COLEF, 2020; Consejo Superior de Deportes-CSD et al., 2020; Fagde, 2020b) no se favoreciera el cambio hacia el consumo virtual, (GrimaldiPuyana et al., 2018) y, por tanto, fuera difícil mantener los hábitos de práctica de AF.

La tendencia ascendente del número de casos después del verano de 2020 hizo necesario nuevas medidas de control de la transmisión de la enfermedad, declarándose el segundo estado de alarma a través del $\mathrm{RD}$ 926/2020, de 25 de octubre (MP, 2020c) y prorrogado hasta el 9 de mayo de 2021 por RD 956/2020, de 3 de noviembre (MP, 2020d), si bien en esta ocasión las medidas eran menos restrictivas, sólo para ciertos establecimientos y actividades, y con flexibilidad y capacidad de modulación por parte de las comunidades autónomas. Esta nueva situación restrictiva ha supuesto que un año después, no se haya alcanzado un nivel de funcionamiento similar al momento previo a la pandemia en algunas de esas actividades (comercios, gimnasios, restaurantes). Muchos negocios han cerrado (INE, 2021b) y se asume que otros muchos no sobrevivirán. En el ámbito de la $\mathrm{AF}$, los gimnasios y los servicios deportivos que han sobrevivido han tenido que ir cerrando y abriendo periódicamente a lo largo de estos meses (Palco23, 2021), con una actividad productiva irregular, a pesar de que los informes indican la baja incidencia del 


\begin{tabular}{|c|c|c|c|c|c|}
\hline Tipo & Intensidad & Frecuencia & Duración & Cantidad total & Modalidad \\
\hline \multirow{3}{*}{$\begin{array}{l}\text { Ejercicios cardio } \\
\text { vasculares }\end{array}$} & Moderada & $=5 \mathrm{~d} / \mathrm{s}$ & $\begin{array}{c}=30 \mathrm{~m} / \mathrm{d} \\
\text { Sesiones de } 10 \text { minutos como mínimo }\end{array}$ & $\begin{array}{c}=150 \mathrm{~m} / \mathrm{s} \\
\text { Para mayores beneficios } \\
\text { incrementar hasta } 300 \mathrm{~m} / \mathrm{s}\end{array}$ & \multirow{3}{*}{$\begin{array}{l}\begin{array}{c}\text { Ejercicios rítmicos y aeróbicos que involucren grandes grupos } \\
\text { musculares }\end{array}\end{array}$} \\
\hline & Vigorosa & $=3 \mathrm{~d} / \mathrm{s}$ & $=20 \mathrm{~m} / \mathrm{d}$ & $\begin{array}{c}=75 \mathrm{~m} / \mathrm{s} \\
\text { Para ser más efectivos } \\
\text { incrementar hasta } 150 \mathrm{~m} / \mathrm{s}\end{array}$ & \\
\hline & $\begin{array}{l}\text { O combinación de } 3-5 \mathrm{~d} / \mathrm{s} \text { de } \\
\text { intensidad moderada y vigorosa }\end{array}$ & & & & \\
\hline $\begin{array}{l}\text { Aptitud muscular } \\
\text { (resistencia y } \\
\text { fuerza musculares } \\
\text { y potencia) }\end{array}$ & Según la experiencia individual & $\begin{array}{l}2-3 \mathrm{~d} / \mathrm{s} \\
\text { preferiblemente } \\
\text { no consecutivos }\end{array}$ & Un intervalo de descanso razonable & $\begin{array}{l}2-4 \text { series con } 8-12 \\
\text { repeticiones } \\
8-10 \text { ejercicios por sesión }\end{array}$ & $\begin{array}{l}\text { Trabajo de cada grupo muscular principal. } \\
\text { Trabajo que afecte a varios grupos musculares simultáneamente. } \\
\text { Subir escaleras, llevar bolsas de comestibles. } \\
\text { Ejercicios neuromotores que incluyan equilibrio, agilidad y } \\
\text { coordinación }\end{array}$ \\
\hline Flexibilidad & $\begin{array}{l}\text { Estirar hasta el punto de sentir } \\
\text { opresión o leve molestia }\end{array}$ & $\begin{array}{c}=2-3 \mathrm{~d} / \mathrm{s} \\
\text { Mejor } \\
\text { diariamente }\end{array}$ & $\begin{array}{c}\text { 30-60 } \mathrm{sg} / \mathrm{ej} \\
\text { En series de 10-30 sg } \\
\text { Ajustar tiempo, duración y repeticiones de } \\
\text { acuerdo con las necesidades individuales }\end{array}$ & 2-4 series & $\begin{array}{c}\text { Ejercicios para cada uno de los grandes músculos / tendones. } \\
\text { También sirven para mejorar la flexibilidad, los ejercicios de } \\
\text { estabilidad postural y equilibrio }\end{array}$ \\
\hline $\begin{array}{l}\text { Entrenamiento } \\
\text { neuromotor o } \\
\text { funcional }\end{array}$ & Sin determinar & $=2-3 \mathrm{~d} / \mathrm{s}$ & $=20-30 \mathrm{~m} / \mathrm{d}$ & $=60 \mathrm{~m} / \mathrm{s}$ & $\begin{array}{l}\text { Implica equilibrio, coordinación, marcha y agilidad. } \\
\text { Tai chi, yoga }\end{array}$ \\
\hline
\end{tabular}

ota $: \mathrm{m}=$ minutos; $\mathrm{d}=$ día $; \mathrm{s}=$ semana; $\mathrm{s} g=$ segundos; ej=ejercicio

COVID-19 en estos servicios y de que las cifras muestran que no son espacios peligrosos para el contagio (Ministerio de Sanidad, 2020b). No obstante, la declaración de la actividad física y deporte como actividad de carácter esencial dado el claro beneficio para la salud (Congreso de los Diputados [BOCG], 2021) contribuirá a que no se tomen medidas tan drásticas en este sector.

El confinamiento, y las circunstancias mencionadas, han podido modificar la intensidad, cantidad y tipología de práctica de AF recomendados por las autoridades sanitarias (Brown et al., 2013; Garber et al., 2011), mermando el estado general de salud de la población, la inmunidad del cuerpo, su estilo y calidad de vida, con el consiguiente gasto derivado de la inactividad física, estimado para el año 2020 en más de 1.800 millones de euros (Jiménez Gutiérrez et al., 2020).

Ahora bien, teniendo en cuenta que en España se han incrementado los ERTEs y el teletrabajo por el confinamiento (Magaz-González \& García-Tascón, 2020), suponiendo una reducción del tiempo dedicado a trabajar, y a desplazarse para ello (lo que redunda en un aumento del tiempo libre disponible) y considerando que el $40 \%$ de los europeos y el $43.8 \%$ de los españoles indican como principal barrera para la práctica de AF es la falta de tiempo (European Commission, 2018; Subdirección General de Estadística y Estudios [SGEE], 2015), también se puede considerar que en este período de confinamiento se han dado las condiciones para superar dicha barrera, para facilitar la progresión gradual de volumen e intensidad, así como de cantidad y calidad de AF que sugieren los expertos para reducir los efectos negativos de la inactividad para la salud (ACSM, 2018; Brown et al., 2013; Garber et al., 2011) y para incluir en el estilo de vida la práctica de AF.

La práctica de AF, enfocada a la mejora de la salud, debe incluir parámetros de frecuencia, duración, intensidad, tipo y cantidad total de AF recomendados por la OMS (2010) y la American College of Sport Medicine [ACSM] (ACSM, 2018), los cuales disminuyen la morbilidad y la mortalidad prematura, mejoran las funciones cardiorespiratorias y musculares, la salud ósea y se reduce el riesgo de enfermedades no transmisibles (ENT) y de depresión. Además, la ACSM informa que hay que realizar ejercicio más allá de las actividades físicas de la vida diaria; considera «que cierta actividad es mejor que ninguna» y señala que si ésta es más, mejor que menos (ACSM, 2018; Garber et al., 2011, p. 1338). Por otra parte, Rutten et al. (2013) indican que los comportamientos activos deben ser simples, fáciles de realizar y fácilmente disponibles. Una adaptación de dichas recomendaciones se observa en la tabla 1 .

Diversos estudios indican que aun cuando se cumplan las pautas de AF de la OMS y la ACSM, estar sentado durante períodos prolongados, puede comprometer la salud metabólica, particularmente la diabetes (Dunstan et al., 2012; Owen et al., 2010) y la salud en general. Así, las últimas recomendaciones de la ACSM aconsejan incluir períodos cortos de tiempo de bipedestación (ej. caminata alrededor del hogar, etc.) o realizar una caminata de dos minutos cada 20 a 30 minutos sentado (Loh et al., 2020) para reducir los efectos adversos de la inactividad física (ACSM, 2018, Dunstan et al., 2012; Garber et al., 2011; Owen et al., 2010; Rutten et al., 2013), considerando, no obstante, la necesidad de aumentar la práctica deAF (ACSM, 2018; Brown et al., 2013; Garber et al., 2011).

Sin duda, no realizar AF y tener actitudes de sedentarismo está asociado con riesgo elevado de mortalidad por enfermedad cardiovascular, empeoramiento de biomarcadores de enfermedad cardiometabólica, aumento de la obesidad, riesgo de padecer cáncer, 
hipertensión, hiperlipidemia, diabetes tipo II, hiperinsulemia y depresión (American College of Sports Medicine [ACSM], 2018; Biswas et al., 2015; Jakicic et al., 2001). En los países desarrollados esta realidad supone una pandemia, convirtiéndola en un problema de interés prioritario para la salud pública (Abellán-Alemán et al., 2014; Donnelly et al., 2009; Jakicic et al., 2001; Saris et al., 2003). La situación de Covid-19 ha podido incrementar estas dolencias y sumarlas a las más relacionadas con el sedentarismo que registra el INE (2020): tensión alta (20\% de la población) y colesterol alto $(18 \%)$.

Por otra parte, la práctica de AF es la variable que mejor predice la calidad de vida (García et al., 2021; Rodríguez et al., 2020). Se la considera estándar de calidad de vida medida por diversas dimensiones de bienestar y satisfacción con la vida; salud, ocio, interacciones sociales... (Eurostat, 2020). También se la asocia a estilos de vida y actitudes saludables (Cubas et al., 2019; Rodríguez et al., 2020), a un uso equilibrado y satisfactorio del tiempo y a la generación de relaciones interpersonales (Eurostat, 2015). Además, como indica Mutz (2021), permanecer activo y practicar AF tiene un efecto amortiguador ante los desafíos de la pandemia. Incluso se ha sugerido que limitar la AF para detener la pandemia podría no ser una medida efectiva (Amekran \& El Hangouche, 2020) debido a su relación con el estilo de vida saludable. Sin embargo, se ha impuesto un cambio brusco en el comportamiento deportivo debido al confinamiento, lo que se relaciona con alteraciones negativas y disminución en el bienestar (Mutz, 2021; Martínezde-Quel, et al. 2021).

Considerando todo lo expuesto, dado que la pandemia ha impuesto limitaciones para evitar la propagación del virus, que ha alterado las condiciones de práctica de AF, y que ésta, a su vez, ha sido un aspecto clave para mantenerse saludable, influyendo en el bienestar físico y mental y el mantenimiento de la calidad de vida durante el encierro, siendo factor determinante de salud, el presente estudio está dirigido a analizar el impacto del confinamiento por Covid-19 en la práctica de AF de acuerdo con los parámetros de AF recomendados por la OMS y la ACSM y, por ende, en la salud y calidad de vida. Este objetivo general se concreta en las siguientes hipótesis de investigación:

H1: Durante el confinamiento se han mantenido la cantidad, la intensidad y el tipo de práctica de AF.

$\mathrm{H} 2$ : Las personas que anteriormente no realizaban $\mathrm{AF}$, durante el confinamiento sí han realizado AF.

$\mathrm{H} 3$ : Las personas que anteriormente al confinamiento realizaban $\mathrm{AF}$, durante el mismo han continuado realizando AF.

H4: Durante el confinamiento ha aumentado la AF suave y moderada practicada, recomendada por la ACSM, permitiendo reducir los efectos adversos de la inactividad física y de estar sentados.

H5: Las personas en situación de ERTE, han aumentado la cantidad de práctica de AF durante el confinamiento.

\section{Material y método}

\section{Diseño del estudio e instrumento}

El trabajo se ha desarrollado utilizando metodología cuantitativa de corte descriptivo, analítico, no correlacional basado en un muestreo no intencional.

Una vez establecidos los objetivos, se elaboró un cuestionario ad hoc que incluyó, además de datos sociodemográficos, aspectos relativos a los hábitos de AF antes y durante el confinamiento provocado por la enfermedad Covid-19.

Para las distintas preguntas, se incluyeron respuestas dicotómicas ( $\mathrm{Sí} / \mathrm{No}$ ), de selección entre varias opciones, o de categorización a través de la escala Likert (1: nada a 5 : mucho), teniendo en cuenta que los ítems tipo Likert suelen utilizarse en puntos impares para evitar respuestas neutras (Pérez et al., 2002).

El instrumento para recoger la información ha sido un formulario aplicado a través de la plataforma Google Formularios, asegurando en todo momento confidencialidad y anonimato además del consentimiento informado sobre la participación en el estudio. Se ha optado por esta opción de recogida de datos porque permite conocer lo que hacen, opinan o piensan los encuestados (Colás \& Buendía, 2012). Además, la administración del cuestionario on line era no solo la mejor opción sino la única para poder obtener la información por el confinamiento. Las preguntas del cuestionario relativas a esta investigación se recogen en el Anexo.

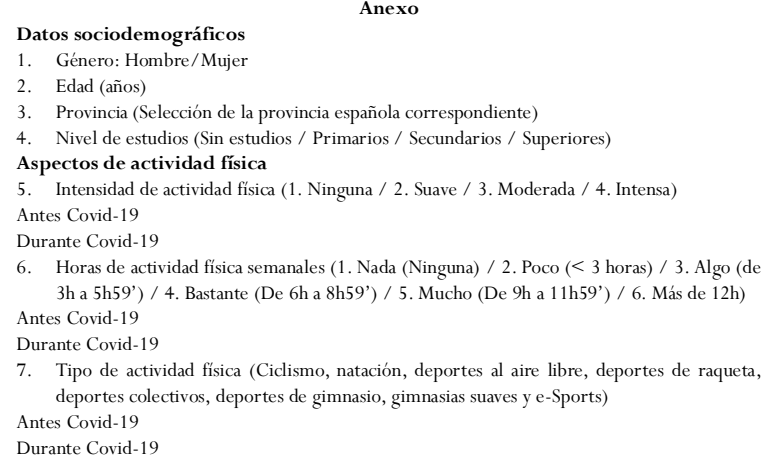


Primeramente, se procedió a validar el contenido del cuestionario a través de juicio de expertos, seleccionados en cumplimiento de los requisitos propuestos por Skjong \& Wentworth (2000). Fue un grupo de 12 expertos en el ámbito de la docencia y de la salud, y participación paritaria en cuanto a género. De ellos, cuatro eran profesores doctores de la universidad en activo con más de 10 años de experiencia en el ámbito de las ciencias de la actividad física y deporte, de psicología y nuevas tecnologías, otros dos de empresas de nuevas tecnologías, dos gestores de servicios municipales y los otros dos expertos, empresarios de centros fitness y gimnasios. También participaron dos personas no expertas en el sector de la AFD, donde una práctica AF de forma habitual, mientras que otra no.

La administración del instrumento o recolección de datos tuvo lugar entre el 10 de abril y el 10 de mayo, periodo de tiempo en el que no se podía realizar $\mathrm{AF}$ al aire libre. El link fue difundido a través de aplicaciones electrónicas, redes sociales y correo electrónico, habiendo contado con la ayuda de asociaciones dando difusión interna y pública del estudio.

\section{Participantes}

El universo de estudio era la población española en general. La muestra obtenida y analizada ha sido de 1046 participantes, de ambos géneros (hombre $48.57 \%$ y mujer $51.43 \%)$ y con una edad media de $40(D T= \pm$ 13.35 años). En relación con la formación, el 81.07\% cuenta con estudios superiores, el $16.63 \%$ tiene estudios secundarios, $2.1 \%$ primarios y únicamente dos casos sin estudios; obteniendo respuestas de casi todas las comunidades autónomas.

\section{Tratamiento de los datos}

Una vez depurada la base de datos, los datos se han tratado con el paquete estadístico SPSS 26.0 (IBM corp., 2019). Primeramente, con el fin de conocer la posibilidad de utilizar técnicas paramétricas para el contraste de las hipótesis propuestas, se sometieron las respuestas obtenidas a la prueba Kolmogorov Smirnov, comprobando que las variables objeto de estudio no cumplían el supuesto de normalidad. Se han utilizado pruebas no paramétricas, donde en vez de aplicar la técnica $t$ de Student (paramétrica), se utiliza la alternativa, la prueba de los rangos de Wilcoxon para muestras relacionadas, estableciendo el nivel de significación en $\mathrm{p}<.5$. Además, se obtienen medidas descriptivas como la media, desviaciones o frecuencias, en función de la naturaleza de los datos.

\section{Resultados}

Con el fin de comprobar el cumplimiento de las recomendaciones de la OMS (2010) y de la ACSM (2018) sobre AF, se han analizado los parámetros de cantidad, intensidad y tipo de AF. En relación con la frecuencia (hábitos diarios/semanales de AF), no se ha considerado oportuno incluir ninguna pregunta al respecto, ya que las condiciones temporales del confinamiento (un periodo muy cor to) no haría factible el análisis de este parámetro. Por otra parte, los resultados relativos a los parámetros cantidad e intensidad se han obtenido y se muestran desde la perspectiva de las categorías utilizadas para la recolección de datos, no en función de valores absolutos.

En la tabla 2 se recoge, para la totalidad de la muestra $(\mathrm{N}=1046)$, la comparación de la cantidad de AF antes $(\mathrm{M}=3.26)$ (categoría 3$)$ y durante el confinamiento $(\mathrm{M}=2.70)$ (categoría 2$)$. Se observa $(Z=$ -13.383, $p$ <.001) que, para el nivel de significación establecido, la cantidad de AF es significativamente menor durante el confinamiento que con anterioridad al mismo. En esa misma tabla se recogen los resultados de comparar la intensidad de AF antes $(\mathrm{M}=3.26)$ y durante $(M=2.76)$ que, para el nivel de significación planteado $(\mathrm{Z}=-15.849, p<.001)$, muestran también una disminución en este parámetro.

Para analizar el tipo de AF, el cuestionario preguntaba por 8 disciplinas deportivas (leyenda tabla 3) valorándose cada una en las categorías recogidas en el Anexo, antes y durante el confinamiento. Para un mejor análisis, en la tabla 3 estas disciplinas se agruparon en los cuatro «tipos» que recomienda la ACSM (2018), expuestos en la tabla 1 .

La figura 1 refleja un aumento durante el confinamiento de los que no han realizado «Nada» de ejercicios cardiovasculares y de aptitud muscular; sin embargo, disminuye los que no han hecho nada de AF de tipo flexibilidad y entrenamiento neuromotor o funcional, notándose este incremento en las categorías de «Poco», «Algo» $\mathrm{y}$ «Bastante».

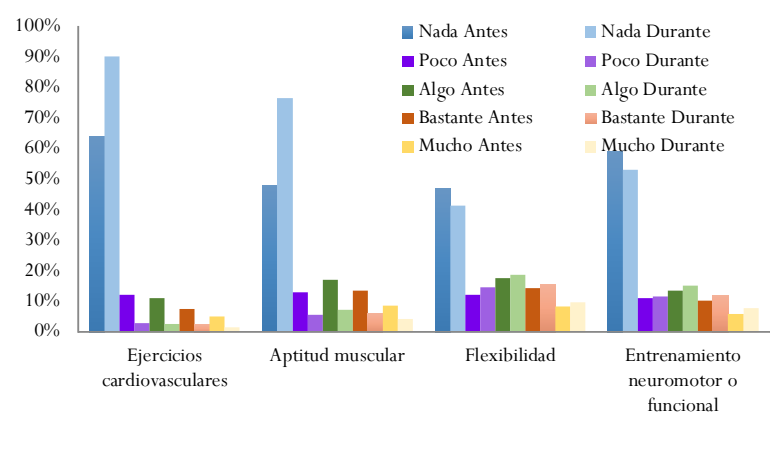

Figura 1. Comparación del tipo de AF practicado antes y durante el confinamiento, en base a la ACSM (2018) 
Tabla 2

\begin{tabular}{lcccccc}
\multicolumn{6}{l}{ Cantidad e Intensidad de AF realizada antes y durante el confinamiento en base a las categorías* } \\
\hline \multirow{2}{*}{$\mathrm{N}=1046$} & Media & Desviación Típica & Wilkoxon W & $\mathrm{Z}$ & \multirow{2}{*}{ Sig. } \\
\hline \multirow{2}{*}{ Cantidad } & Antes & 3.26 & 1.256 & \multirow{2}{*}{52558.000} & -13.383 & \multirow{2}{*}{.000} \\
& Durante & 2.70 & 1.095 & & \\
\hline \multirow{2}{*}{ Intensidad } & Antes & 3.26 & .811 & \multirow{2}{*}{26011.500} & -15.849 & \multirow{2}{*}{000} \\
& Durante & 2.76 & .879 & & & \\
\hline
\end{tabular}

Nota: $\mathrm{N}=$ Tamaño de la muestra. $\mathrm{Z}=$ Puntuación Z. Sig. $=\mathrm{p}$-valor

*Categorías Cantidad: Horas AF semanal (1. Nada (Ninguna) / 2. Poco $(<3$ horas) / 3. Algo (de 3 h a 5 h59') / 4. Bastante (De 6h a 8 h59') / 5. Mucho (De 9h a 11h59') / 6. Más de 12h). Categorías Intensidad: 1. Ninguna / 2. Suave / 3. Moderada / 4. Intensa

Tabla 3

Comparativa de tipo de AF practicada antes y durante el confinamiento (ACSM, 2018) (porcentajes) $\begin{array}{ccccc}\text { Nada } & \text { Poco } & \text { Algo } & \text { Bastante } & \text { Mucho } \\ \text { Antes Durante AntesDurante Antes } & \text { Durante Antes Durante } & \text { Antes Durante }\end{array}$

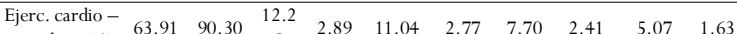

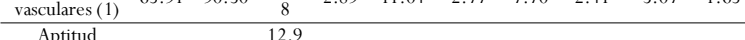
\begin{tabular}{lllllllllll}
47.80 & 76.56 & 12.9 & 5.74 & 17.00 & 7.23 & 13.73 & 6.27 & 8.55 & 4.21 \\
\hline
\end{tabular} \begin{tabular}{lllllllllll}
\hline Flexibilidad (3) & 47.23 & 41.44 & 12.2 & 14.72 & 17.69 & 18.79 & 14.34 & 15.39 & 8.46 & 9.66
\end{tabular} \begin{tabular}{lllllllllll}
\hline Entrenamiento & 11.0 & & & & &
\end{tabular} $\begin{array}{lllclllllll}\text { neuromotor o } & 59.24 & 53.22 & 6 & 11.73 & 13.48 & 15.17 & 10.26 & 11.95 & 5.96 & 7.93\end{array}$ funcional (4)

Nota: (1) ciclismo, natación, deportes de raqueta, deportes colectivos; (2) ciclismo, deportes a aire libre, deportes de raqueta, deportes colectivos, deportes de gimnasio; (3) deportes de gimnasio, gimnasias suaves; (4) deportes de gimnasio, gimnasias suaves y e-Sports

Tabla 4

Sujetos que no realizan $A F$ antes $y$ durante el confinamiento

\begin{tabular}{|c|c|c|c|c|c|c|}
\hline $\mathrm{N}=54$ & & Media & Desviación Típica & Wilkoxon W & $\mathrm{Z}$ & Sig. \\
\hline \multirow[b]{2}{*}{ Cantidad } & Antes & 1.00 & .00 & \multirow[b]{2}{*}{378.000} & \multirow{2}{*}{4.724} & \multirow[b]{2}{*}{.000} \\
\hline & Durante & 1.70 & .80 & & & \\
\hline \multirow{2}{*}{ Intensidad } & Antes & 1.00 & .00 & \multirow{2}{*}{269.000} & \multirow{2}{*}{2.479} & \multirow{2}{*}{.013} \\
\hline & Durante & 2.00 & .90 & & & \\
\hline
\end{tabular}

Nota: $\mathrm{N}=$ Tamaño de la muestra. $\mathrm{Z}=$ Puntuación $\mathrm{Z}$. Sig. =p-valor

*Categorías Cantidad: Horas AF semanal (1. Nada (Ninguna) / 2. Poco ( $<3$ horas) / 3. Algo (de 3h a 5 h59') / 4. Bastante (De 6h a 8h59') / 5. Mucho (De 9h a 11h59') / 6. Más de 12h). Categorías Intensidad: 1. Ninguna / 2. Suave / 3. Moderada / 4. Intensa

Tabla 5 \begin{tabular}{llllll} 
Cantidad e intensidad de AF antes $y$ durante en sujetos que no realizan $A F$ durante confinamiento & \\
\hline $\mathrm{N}=115$ & Media & Desviación Típica & Wilkoxon W & $\mathrm{Z}$ & Sig. \\
\hline
\end{tabular} \begin{tabular}{ccccccc}
\hline \multirow{2}{*}{ Cantidad } & Antes & 2.40 & 1.18 & 235.243 & -8.323 & .000 \\
\cline { 2 - 5 } & Durante & 1.00 & 00 & 2400 & & \\
\hline
\end{tabular}

\begin{tabular}{cccccc}
\hline Intensidad Antes & 2.60 & .99 & 211.450 & -7.940 & .000
\end{tabular}

Nota: $\mathrm{N}=$ Tama ̃o de la muestra $\mathrm{Z}=\mathrm{P}$.00

Nete $3 \mathrm{~h}$ a $\left.5 \mathrm{~h} 59^{\prime}\right) / 4$. Bastante (De 6h a $\left.8 \mathrm{~h} 59^{\prime}\right) / 5$. Mucho (De $9 \mathrm{~h}$ a $\left.11 \mathrm{~h} 59^{\prime}\right) / 6$. Más de 12h). Categorías Intensidad: 1. Ninguna / 2. Suave / 3. Moderada / 4. Intensa

Tabla 6

Cantidad de AF realizada antes y durante por personas en situación de ERTE durante el confinamiento \begin{tabular}{cccccc}
$\mathrm{N}=115$ & Media & Desviación Típica & Wilkoxon W & Z & Sig. \\
\hline
\end{tabular} \begin{tabular}{lcccccc}
\hline Cantidad & Antes & 3.66 & 1.39 & 320.000 & -5.640 & .000
\end{tabular} Nota: $\mathrm{N}=$ Tamaño de la muestra. $\mathrm{Z}=$ Puntuación $\mathrm{Z}$. Sig. $=\mathrm{p}$-valor

*Categorías Cantidad. Horas AF semanal (1. Nada (Ninguna) / 2. Poco $(<3$ horas) / 3. Aloo (de $3 \mathrm{~h}$ a $\left.5 \mathrm{~h} 59^{\prime}\right) / 4$. Bastante (De $6 \mathrm{~h}$ a $\left.8 \mathrm{~h}^{\prime} 9^{\prime}\right) / 5$. Mucho (De $9 \mathrm{~h}$ a $\left.11 \mathrm{~h} 59^{\prime}\right) / 6$. Más de $12 \mathrm{~h}$ ). Categorías Intensidad: 1. Ninguna / 2. Suave / 3. Moderada / 4. Intensa

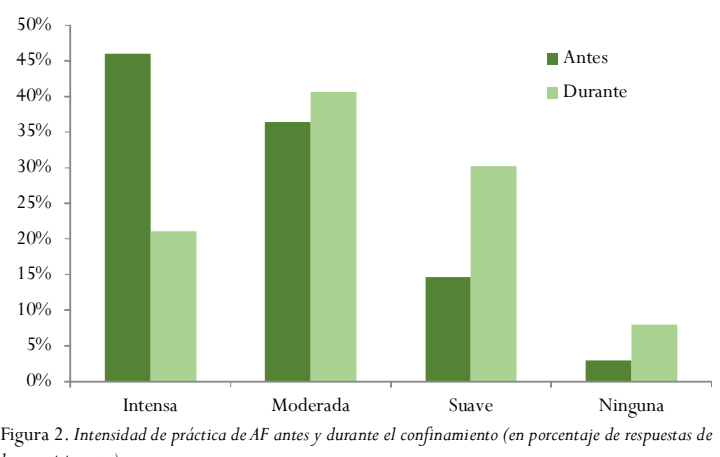
los participantes)

Los resultados recogidos en la tabla 4, consideran únicamente aquellos sujetos que antes del confinamiento no realizaban AF $(\mathrm{N}=54)$ y comparando con su situación durante en relación con cantidad, muestran que de realizar «Nada» de AF han pasado a realizar «Algo» de AF $(M=1.70)$ situándose en la categoría que realiza AF entre 0 y 3 horas semanales. Respecto al parámetro intensidad, los resultados reflejan también un aumento desde «Ninguna» a intensidad «Suave» $(\mathrm{M}=2.00)$.

La tabla 5 recoge los resultados relativos a aquellas personas ( $\mathrm{N}=115,11.6 \%)$ que con anterioridad realizaban AF e indicaron no realizar ningún tipo de AF durante la pandemia Covid-19. En cuanto a cantidad, este subgrupo practicaba antes del confinamiento en un rango variable de horas (de $3 \mathrm{~h}$ a más de $9 \mathrm{~h}$ semanales), siendo la media $\mathrm{M}=2.40$ y la desviación típica $s=$ 1.18. La intensidad de AF de este subgrupo, antes del confinamiento era tanto «Suave», como «Moderada» e «Intensa» $(\mathrm{M}=2.60)$ con una desviación típica $s=.99, \mathrm{y}$ ahora es $M=1.00$. En la figura 2 se observa que entre los practicantes ha descendido la intensidad, pasando a AF «Moderada»y «Suave», principalmente.

En la tabla 6 se muestra la comparativa de resultados de los individuos $(\mathrm{N}=95)$ en situación de ERTE durante el confinamiento $(Z=-5.640, p<.001)$. Se observa un descenso significativo en el parámetro cantidad (durante) con $\mathrm{M}=2.80$, en relación con las horas dedicadas antes del confinamiento $(M=3.70)$ en situación laboral normal.

\section{Discusión}

La declaración del estado de alarma sanitaria en España en 2020, y el confinamiento al que fue sometida la población, supuso un claro descenso de práctica de AF (López-Bueno et al., 2020). A pesar de las recomendaciones de asociaciones profesionales (COLEF, 2020; CSD \& Consejo-COLEF, 2020) o expertos en AFD (Márquez, 2020; Mera et al. 2020; Serrano, 2020), de las múltiples actividades virtuales existentes, y de que los medios de comunicación afirmaron insistentemente que la práctica de AF se había convertido en una de las actividades rutinarias entre las personas confinadas, los datos no confirman esa realidad. La reducción de la práctica de AF es drástica en este período, como también confirman los estudios publicados en poblaciones universitarias (Astiazarán \& Ara, 2020), o en la población en conjunto (Balluerka et al., 2020). Si bien otras investigaciones concluyen lo contrario (IBV, 2020), sus autores reconocen trabajar con una muestra especialmente activa.

Recordando el objetivo general de este estudio, dirigido a conocer hasta qué punto se vieron afectados los parámetros de AF recomendados por la ACSM y la OMS, se analizaron cantidad, intensidad y tipo de AF realizada durante el confinamiento.

Atendiendo a la H1, se observa que, para el nivel de 
significación establecido, la cantidad de AF es menor durante el confinamiento que con anterioridad al mismo. Los resultados obtenidos relacionados con la cantidad ponen de manifiesto una disminución significativa $(Z=$ -13.383, $p<.001)$, pasando de 3h-6h semanales $(\mathrm{M}=$ 3.26) a menos de $3 \mathrm{~h}$ semanales $(\mathrm{M}=2.70)$, coincidiendo con los estudios ya mencionados, aunque no con el del IBV (2020). Además, el aumento del «tiempo libre» durante el confinamiento no produjo un aumento del tiempo dedicado a realizar AF, aun siendo la falta de tiempo la principal barrera para practicar AF (European Commission, 2018; Ministerio de Educación C. y D., 2020; SGEE, 2015).

Respecto a la intensidad, los datos demuestran una disminución de este parámetro, pasando de $\mathrm{M}=3.26$ antes a $\mathrm{M}=2.76$ durante el periodo analizado, lo que, para el nivel de significación planteado $(Z=-15.849$, y $p<.001)$ confirman también una disminución significativa en la intensidad, en consonancia con el trabajo del IBV (2020). Este resultado puede considerarse normal debido a la dificultad o imposibilidad para realizar muchas modalidades de AF (por limitaciones de espacio, equipamiento, materiales...) suponiendo para la práctica deportiva habitual, la adaptación o el cambio.

En cuanto a la disciplina deportiva, durante el confinamiento se han remplazado los deportes al aire libre, de gimnasio, ciclismo y colectivos (ejercicios «cardiovasculares» y de «aptitud muscular», tabla 1) por prácticas fáciles realizadas en el hogar, con pocas demandas de espacio, equipamiento y material, como fitness y musculación (deportes de gimnasio), yoga y Pilates (gimnasias suaves), ciclismo (estático) y e-Sports (IBV, 2020; Urban Sports Club, 2020). Esta modificación de disciplinas deportivas, espacio y/o material han podido influir en que intensidad y cantidad hayan sido inferiores a las recomendaciones de expertos; resultados coincidentes con otras investigaciones (Astiazarán \& Ara, 2020; Fitbit Staff, 2020; IBV, 2020).

Según Magaz-González y García-Tascón (2020), en la elección de dichas disciplinas deportivas ha podido influir la oferta virtual de AF existente en plataformas virtuales y redes sociales, de forma que la AF virtual se ha comportado como una opción muy positiva para ayudar a practicar en el hogar las modalidades recomendadas por la ACSM y la OMS. A los estudios que alertan de la inactividad y sus efectos adversos (ACSM, 2018; Dunstan et al., 2012; Garber et al., 2011; Owen et al., 2010; Rutten et al., 2013), se añade este período de confinamiento, y aunque se han realizado algunos tipos de AF (ejercicios de flexibilidad y entrenamiento neuromotor), es preocupante que hayan sido más los que no han realizado nada de ejercicio cardiovascular y de aptitud muscular, contraviniendo las recomendaciones de los expertos (ACSM, 2018; OMS, 2010).

En resumen, en cuanto a la $\mathrm{H} 1$, no se puede afirmar que se hayan mantenido ni la cantidad ni la intensidad de práctica de AF, y tampoco se han seguido las recomendaciones indicadas por la ACSM y la OMS. Respecto al tipo de práctica de AF, se acepta, pero con reserva.

Se acepta la $\mathrm{H} 2$; y aunque son pocos los encuestados que manifestaron no hacer «Nada» de AF antes del confinamiento $(5.1 \%)$, es positivo observar un aumento en la cantidad (menos de 3 horas) y la intensidad (suave) de práctica de AF realizada durante el confinamiento por este subgrupo, porcentaje similar al estudio del IBV (2020). Campañas como «Muévete en casa» (RTVE, 2020) o la del Consejo-COLEF (2020) han podido estimular a dichas personas a practicar AF y con ello mejorar su estado general de salud. La $\mathrm{H} 3$ se rechaza, ya que $11.6 \%$ de personas que practicaban antes, no han continuado realizando AF durante el confinamiento.

Considerando que es mejor hacer cierta AF que ninguna (Garber et al., 2011) y que es preferible hacer AF suave y ejercicios simples a estar demasiado tiempo inactivo (ACSM, 2018; Dunstan et al., 2012; Rutten et al., 2013), se puede afirmar que entre los que han practicado AF durante el confinamiento, aunque ha disminuido la práctica intensa, ha aumentado la práctica «moderada»y «suave» que permite reducir los efectos adversos del sedentarismo, aceptándose la H4.

Analizando la muestra en situación de ERTE (personas con mayor tiempo libre y sin barreras para practicar AF a causa de la falta de tiempo a priori), aunque sería de esperar un aumento de práctica por este colectivo, los datos muestran no solo que la cantidad de AF es menor (pasa de 3.66 a 2.76; $p<.001$ ) sino que es muy inferior a la de la muestra general (de 3.26 a 2.70; $p<$ .001), rechazándose por tanto la H5. Este hecho puede suponer que la falta de tiempo no es realmente la principal barrera para no practicar AF y deberían considerarse otras variables relevantes, por ejemplo, el cuidado de menores y familiares, la realización de otras actividades, el estado de ánimo, la falta de costumbre, lesiones y/o dolores, entre otras, y que han podido ser clave para mantenerse inactivo. Además de éstas, otros estudios indican como principales bloqueadores de práctica la motivación o no gustar practicar (European Commission, 2018; SGEE, 2015), o la falta de socialización y contacto (IBV, 2020). 


\section{Conclusiones}

La pandemia por Covid-19 y el subsiguiente aislamiento ha impactado sobre la práctica de $\mathrm{AF}$, viéndose afectada la calidad de vida y la salud de los españoles. Pero también es una oportunidad única para estudiar los efectos de una modificación de las condiciones de práctica de AF en la salud, la calidad de vida y el bienestar.

Como reconoce el Consejo de Europa (Diario Oficial del Consejo Unión Europea, 2020), el deporte es fundamental para contribuir al bienestar de la salud del ciudadano. Los representantes de los Gobiernos de los Estados miembros han indicado que la pandemia por Covid-19 ha tenido consecuencias devastadoras para el deporte e instan a adoptar medidas en diferentes niveles (nacional y especialmente local), para su recuperación, pues es una herramienta que ayuda al desarrollo económico y social hacia unas comunidades más saludables y activas (Diario Oficial del Consejo Unión Europea, 2020). Así ha sucedido en España al declararse la AF como actividad esencial en situaciones de pandemia (BOCG, 2021). También la Unesco (2021) hace un llamamiento a la inversión en educación física de calidad para apoyar la recuperación post-Covid-19 (física, mental, social y económica).

La evidencia científica es clara, la práctica regular de deporte y ejercicio es «la poli-píldora más eficaz y eficiente para construir una sociedad más capaz, más feliz, más sostenible» (Jiménez-Gutiérrez et al., 2020) $\mathrm{y}$ una de las dimensiones que contribuye al bienestar y la calidad de vida (Eurostat, 2015). Siendo un instrumento clave de promoción y prevención de la salud, de ahorro en costes socio-sanitarios, de activación social y económica, de desarrollo sostenible y conservación del planeta (Guthold et al., 2018; Peake et al., 2017; Reimers et al., 2012).

Sin duda, el confinamiento ha variado las condiciones de práctica de AF, afectando a cantidad e intensidad (que han disminuido), al tipo AF (pasando a realizarse sólo actividades de entrenamiento neuromotor o funcional y de flexibilidad) y a las disciplinas deportivas practicadas (de fácil adaptación al hogar y poca demanda de espacio y equipamiento). Este descenso se ha producido a pesar del diseño de programas de AF ad hoc y de la oferta en entornos virtuales, debiéndose además a factores de disponibilidad de material o instalaciones específicas, situaciones familiares, etc., o la accesibilidad y utilidad percibida de las tecnologías aplicadas a la AF.
Aunque el confinamiento ha evidenciado el abandono de la práctica de AF por un $5.46 \%$ más de la muestra, por lo menos, entre los practicantes se ha pasado de la $\mathrm{AF}$ «Intensa» a «Moderada y Suave», suficiente para contrarrestar los efectos negativos de permanecer mucho tiempo sentado delante del ordenador o tumbado frente a la televisión.

Además, la barrera para practicar $\mathrm{AF}$ «falta de tiempo» no es bloqueadora de práctica, pues el tiempo dedicado a trabajar de muchas personas ha estado disponible. Asimismo, se considera al subgrupo que antes no hacía nada y que durante realizó AF «Suave», como población sensible a las campañas de promoción de la AF y sería oportuno que las instituciones enfocaran algunas de sus estrategias a estas personas que se han animado a comenzar a realizar AF durante el confinamiento.

Todos los resultados avalan que durante el confinamiento han peligrado o desaparecido las condiciones que permiten mantener los estándares de calidad de vida (práctica de AF bajo parámetros de salud y, especialmente, los relacionados con la práctica de $\mathrm{AF}$ en interacción social). De esta manera, la inactividad física durante este periodo ha frenado la construcción de una sociedad más capaz, feliz y sostenible.

Este confinamiento y los meses sucesivos han mostrado la reducción de la práctica de $\mathrm{AF}$ tanto en intensidad como en tiempo (Astiazarán \& Ara, 2020; Balluerka et al., 2020; Fitbit Staff, 2020; Magaz-González \& GarcíaTascón, 2020). Esta situación no sólo debe revertirse, como así insta la Unesco (2021), sino que además se deben incrementar los valores de práctica previos a la pandemia, puesto que, tan solo 15 minutos de ejercicio físico al día se puede aumentar la esperanza de vida tres años y reducir un 14\% la mortalidad (Harvard, 2013).

La pandemia ha afectado al modelo de negocio del sector de la AF. Ha hecho necesario, primeramente, diseñar estrategias para recuperar la confianza del cliente para volver a los gimnasios (Prado et al., 2020) garantizando medidas sanitarias de ventilación, distanciamiento, etc. y seguidamente explorar la gama de los servicios on line y en streaming para mantener o aumentar las pautas de AF recomendadas (Porras-Hilarión et al., 2021).

Para finalizar, indicar que los resultados mostrados en esta investigación tienen el ánimo de informar de la necesidad de profundizar en estudios para el diseño de nuevas estrategias de adherencia, fidelización y hábitos de práctica de AF saludable en situación de pandemia y post pandemia, en las que, factores psicológicos, económicos y/o laborales van a influir en la fluctuación de la 
práctica de AF.

\section{Referencias}

Abellán-Alemán, J., Sainz de Baranda Andujar, P., \& OrtínOrtín, E. J. (2014). Guía para la prescripción de ejercicio físico en pacientes con riesgo cardiovascular. Recuperado de https: / /www.seh-lelha.org/wp-content/uploads / 2017/03/GuiaEjercicioRCV.pdf

ACSM. (2018). ACSM's guidelines for exercise testing and prescription (D. Riebe (ed.); Thent). Wolters Kluwer Health.

Alarcón-Meza, E. I., \& Hall-López, J. A. (2021). Actividad física en estudiantes deportistas universitarios, previo y en el confinamiento por pandemia asociada al COVID-19. Retos, 39, 572-575. https://doi.org/10.47197/ retos.v0i39.81293

Amekran,Y., \& El Hangouche,A. J. (2020). Coronavirus disease (COVID-19) and the need to maintain regular physical activity. The Journal of Sports Medicine and Physical Fitness, 61(1), 159-160. https://doi.org/10.23736/S00224707.20.11524-X

Arras-Vota, A., Bordas-Beltrán, J., Mondaca-Fernández, F., \& Rivera-Sosa, J. (2020). El caso sede México: Formación en Educación Física en e-entornos universitarios durante la contingencia de la COVID-19 (The México heartquarters case: Physical Education Teacher Education in e-university environments during the COVID-19 contingency). Retos, 41, 35-46. https://doi.org/ $10.47197 /$ retos.v0i41.83529

Astiazarán, J., \& Ara, I. (coord.). (2020). Actividad Física en la población universitaria durante el confinamiento por COVID19: determinantes y consecuencias sobre el estado de salud y calidad de vida. Recuperado de https: / / www.csd.gob.es/ sites / default / files / media / files / 2020-06/ Resumen\%20Ejecutivo\%20Proyecto\%20AF\%20confinam iento\%20universitarios\%20200605_FINAL\%20...pdf

Baena-Morales, S., López-Morales, J., \& García-Taibo, O. (2021). La intervención docente en educación física durante el periodo de cuarentena por COVID-19. Retos, 39, 388-395. https://doi.org/10.47197/ retos.v0i39.80089

Balluerka, N., Gómez, J., Hidalgo, M. D., Gorostiaga, A., Espada, J. P., Padilla, J. L., \& Santed, M. Á. (2020). Las consecuencias psicológicas de la COVID-19 y el confinamiento. Informe de investigación. Recuperado de https:// canal.ugr.es/wp-content/uploads/2020/05/Consecuencias-psicologicas-COVID-19.pdf

Banco de España. (2020). Proyecciones macroeconómicas. Análisis Económico. Recuperado de https://www.bde.es/ bde/es/areas/analisis-economi/analisis-economi/proyecciones-mac/Proyecciones_macroeconomicas.html Bosch, J., García, J., \& Murillo, C. (2020). El sector del deporte: un paciente económico del coronavirus con un diagnóstico muy incierto a fecha de hoy - SEED. SEED. Deporte. Recupera- do de https://seed-deporte.es/el-sector-del-deporteun-paciente-economico-del-coronavirus-con-un-diagnostico-muy-incierto-a-fecha-de-hoy/

Castañeda-Babarro, A., Arbillaga-Etxarri, A., GutiérrezSantamaría, B., \& Coca,A. (2020). PhysicalActivity Change during COVID-19 Confinement. International Journal of Environmental Research and Public Health, 17(18), 1-10. https: / / doi.org/10.3390/ijerph17186878

Colás, M. P. \& Buendía, L. (2012). Investigación Educativa. Sevilla: Alfar.

COLEF. (2020). Recomendaciones docentes para una Educación Física segura y responsable ante la nueva normalidad. Recuperado de https://www.consejo-colef.es/post/covid19. guia-ef-nueva-normalidad

Congreso de los Diputados. (2021). Proposición no de Ley $161 / 001821$ relativa a la urgente necesidad de declarar la actividad física y deporte como actividad esencial tras la crisis sanitaria ocasionada por el COVID-19. BOCG, núm. 241, de 17 de marzo de 2021, 63-65.

Consejo-COLEF. (2020). \#YoMeMuevoEnCasa, campaña del Consejo COLEF durante el Estado de Alarma. Recuperado de https: / / www.consejo-colef.es/post/covid19-vgambau CSD \& Consejo-COLEF. (2020). YoMeMuevoEnCasa. Últimas Noticias. Recuperado de https://www.csd.gob.es/es/ la-campana-yomemuevoencasa-del-csd-y-el-consejocolef-recuerda-la-importancia-de-mantener-un-estilode-vida-activo-durante

Cubas-Martinez, V., Marco-Ahulló, A., Monfort-Torres, G., Villarrasa-Sapiña, I., Pardo-Ibañez, A., Garcia-Masso, X. (2019). Perfiles de actividad física, obesidad, autoestima y relaciones sociales del alumnado de primaria: un estudio piloto con Self-Organizing Maps. Retos, 36, 146-151. https: / /doi.org/10.47197/retos.v36i36.67549

Diario Oficial del Consejo Unión Europea. (2020). Conclusiones del Consejo y de los Representantes de los Gobiernos de los Estados miembros, reunidos en el seno del Consejo, relativas a las repercusiones de la pandemia de COVID-19 y la recuperación del sector del deporte. Recuperado de https:// www.boe.es/doue/2020/214/Y00001-00004.pdf

Donnelly, J., Blair, S., Jakicic, J., Manore, M., Rankin, J., \& Smith, B. (2009). Appropriate Physical Activity Intervention Strategies forWeight Loss and Prevention of Weight Regain for Adults. Medicine and Science in Sports and Exercise, 41 (2), 459-471. https: / / doi.org/10.1249/ MSS.0b013e3181949333

Dunstan, D.W., Howard, B., Healy, G. N., \& Owen, N. (2012). Too much sitting - A health hazard. Diabetes Research and Clinical Practice, 97(3), 368-376. https://doi.org/ 10.1016/j.diabres.2012.05.020

European Commission. (2018). Special Eurobarometer 472, sport and physical activity. Recuperado de https:// data.europa.eu/euodp/en / data / dataset / S2164_88_4_472_ENG

Eurostat. (2015). Quality of life. Facts and views. Luxembourg: 
Publications Office of the European Union. https: / / doi.org/10.2785/59737

Eurostat. (2020). Quality of life indicators - health. Eurostat. Statistic Explained. Recuperado de https:// ec.europa.eu/eurostat/statistics-explained/ index.php?title=Quality_of_life_indicators__health\#Europeans_live_longer_and_healthier_lives

Fagde. (2020a). Mientras dure el confinamiento por el COVID-19, \#YoEntrenoEnCasa. FAGDE. Recuperado de https:// www.fagde.org/es/articulo/195/mientras-dure-elconfinamiento-por-el-covid-19-yoentrenoencasa/

Fagde. (2020b). Go Fit everywhere, la alternativa tras el cierre de todos los centros de la cadena por el coronavirus. 20 Noticias. FAGDE, Noticias. Recuperado de https:// www.fagde.org/es/post/785/go-fit-everywhere-la-alternativa-tras-el-cierre-de-todos-los-centros-de-la-cadena-por-el-coronavirus/

Fitbit Staff. (2020). The Impact Of Coronavirus On Global Activity. Fitbit News. Recuperado de https://blog.fitbit.com/ covid-19-global-activity/

Frontiers. (2020). Coronavirus Disease (COVID-19): Psychoeducational Variables Involved in the Health Emergency | Frontiers Research Topic. Frontiers. Recuperado de https:/ /www.frontiersin.org/research-topics/13709/ coronavirus-disease-covid-19-psychoeducational-variab le s - involved - i n - the - h e a l th emergency?utm_campaign $=$ sub-cov-rt-fpsyg-

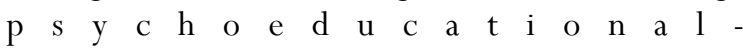
variables\&utm_medium $=$ cvlp\&utm_source $=$ fweb

Garber, C., Blissmer, B., Deschenes, M., Franklin, B., Lamonte, M., Lee, I.-M., Nieman, D., \& Swain, D. (2011). Quantity and quality of exercise for developing and maintaining cardiorespiratory, musculoskeletal, and neuromotor fitness in apparently healthy adults: guidance for prescribing exercise. Medicine and Science in Sports and Exercise, 43(7), 1334-1359. https://doi.org/10.1249/ MSS.0b013e318213fefb

García, E., Canto, Rosa, A., \& Nieto, L. (2021). Nivel de actividad física, consumo habitual de tabaco y alcohol, y su relación con la calidad de vida en adolescentes españoles. Retos, 39, 112-119. https://doi.org/10.47197/ retos.v0i39.78489

García-Tascón, M. (2020). COVID-19 y el otro gran invisible del deporte:el equipamiento deportivo. FAGDE, Deporte y Covid19. Recuperado de https://www.fagde.org/es/post/ 878/ covid-19-y-el-otro-gran-invisible-del-deporte-elequipamiento-deportivo/

Grimaldi-Puyana, M., Sánchez-Oliver, J. A., \& GarcíaFernández, J. (2018). Management Organizations of Spanish Gyms. Apunts. Educación Física y Deportes, 3(133), 7-19. Recuperado de https: / / www.raco.cat/index.php/ ApuntsEFD/article/view/340449

Guthold, R., Stevens, G. A., Riley, L. M., \& Bull, F. C. (2018). Worldwide trends in insufficient physical activity from
2001 to 2016: a pooled analysis of 358 population-based surveys with 1.9 million participants. The Lancet. Global Health, 6(10), e1077-e1086. https: / / doi.org/10.1016/ S2214-109X(18)30357-7

Harvard. (2013). Exercise: 15 minutes a day ups lifespan by 3 years. Exercise: a prescription for making your heart stronger. Harvard heart letter: from Harvard Medical School, 24(4). Recuperado de https:// www.health.harvard.edu/heart-health/exercise-15minutes-a-day-ups-lifespan-by-3-years

Hortigüela-Alcalá, D., Garijo, A., \& Pérez-Pueyo, Á. (2021). La Educación Física en el contexto COVID-19. Un relato de profesores de diferentes etapas educativas. Retos, 41 , 764-774. https: / / doi.org/10.47197/ retos.v41i0.86368

IBM Corp. (2019). IBM SPSS Statistics forWindows, Version 26.0. Armonk, NY: IBM Corp. Recuperado de https:// www.ibm.com/support/pages/how-cite-ibm-spssstatistics-or-earlier-versions-spss

IBV. (2020). Actividad Física en tiempos COVID-19. Informe de Resultados. Recuperado de http://munideporte.com/ imagenes/documentacion/ficheros/03E3FDFD.pdf

INE - Instituto Nacional de Estadística. (2020). Anuario Estadístico de España. Recuperado de https://www.ine.es/ prodyser/pubweb/anuario20/anu20_completo.pdf

INE - Instituto Nacional de Estadística. (14 de abril de 2021a). Crecimiento en volumen. (Revisión estadística 2019) [sección prensa]. Recuperado de https://www.ine.es/prensa/ pib_tabla_cne.htm

INE - Instituto Nacional de Estadística. (2021b). Estadística experimental - Coyuntura demográfica de empresas - Primer, segundo y tercer trimestre de 2020. Recuperado de https: / / www.ine.es/prensa/experimental_codem.pdf

IOC. (2020). Joint Statement from the International Olympic Committee and theTokyo 2020 organising committee. IOC News. Recuperado de https://www.olympic.org/ news/joint-statement-from-the-international-olympiccommittee-and-the-tokyo-2020-organising-committee

Jakicic, J. M., Clark, K., Coleman, E., Donnelly, J. E., Foreyt, J., Melanson, E., Volek, J., \&Volpe, S. L. (2001).Appropriate intervention strategies for weight loss and prevention of weight regain for adults. Medicine \& Science in Sports \& Exercise, 33(12). https://doi.org/10.1097/00005768200112000-00026

Jiménez-Barreto, I. \& Borges, P. J. (2021). Efecto de un programa de entrenamiento en casa mediado por ZOOM, en fútbol femenino durante la pandemia Covid-19, en la percepción subjetiva del esfuerzo y el apoyo a las necesidades psicológicas básicas. Retos, 41, 616-627. https: / / doi.org/10.47197/retos.v0i41.82481

Jiménez-Gutiérrez, A., Mayo-Mauriz, X., López-Valenciano, A., \& Ardanuy-Pizarro, M. (2020). Estudio de Evaluacion del Impacto de la pandemia del COVID-19 sobre el Ecosistema del Deporte en Espana. Recuperado de https:// 
deporteespana.es/es/noticia/4985/-estudio-del-impacto-del-covid-19-sobre-el-ecosistema-del-deporteespanol-documento-completo/

Loh, R., Stamatakis, E., Folkerts, D. et al. (2020). Effects of Interrupting Prolonged Sitting with Physical Activity Breaks on Blood Glucose, Insulin and Triacylglycerol Measures: A Systematic Review and Meta-analysis. Sports Medicine, 50, 295-330 https://doi.org/10.1007/ s40279-019-01183-w

López, P. (2020a). Administración y gimnasios buscan fórmulas para compensar el cierre de centros deportivos. Palco23. Recuperado de https://www.palco23.com/fitness/ administracion-y-gimnasios-buscan-formulas-para-compensar-el-cierre-de-centros-deportivos.html

López, P. (2020b). De secundario a 'mainstream': las cadenas de gimnasios abrazan el entrenamiento virtual por el Covid-19. Palco23. Recuperado de https://www.palco23.com/ fitness/de-secundario-a-mainstream-las-cadenas-degimnasios-abrazan-el-entrenamiento-virtual-por-elcovid-19.html

López-Bueno, R., Calatayud, J., Andersen, L. L., BalsalobreFernández, C., Casaña, J., Casajús, J.A., Smith, L., \& LópezSánchez, G.F. (2020). Immediate Impact of the COVID19 Confinement on Physical Activity Levels in Spanish Adults. Sustainability, 12, 1-10. https://doi.org/ $10.3390 /$ su12145708

Magaz-González, A. M., \& García-Tascón, M. (2020). Resultados preliminares del «Estudio sobre el confinamiento en relación con la actividad física y el uso de las TIC». SEED, Opinión. Recuperado de https://seed-deporte.es/resultados-preliminares-del-estudio-sobre-el-confinamientoen-relacion-con-la-actividad-fisica-y-el-uso-de-las-tic/

Márquez, J. J. (2020). Inactividad física, ejercicio y pandemia COVID-19. Viref. Revista de Educación Física, 9(2), 43-56. Recuperado de https://revistas.udea.edu.co/ index.php/viref/article/view/342196/20802578

Martín, L. (2020). España roza los cuatro millones de afectados por ERTEs. Economía Digital. Recuperado de https:// www.economiadigital.es/directivos-y-empresas/espanaroza-los-4-millones-de-afectados-por-ertes-porcoronavirus_20053920_102.html

Martínez-de-Quel, O., Suárez-Iglesias, D. , López-Flores, M., \& Ayán Pérez, C. (2021). Physical activity, dietary habits and sleep quality before and during COVID-19 lockdown: A longitudinal study. Appetite, 158, 1-6. https: / / doi.org/ 10.1016/j.appet.2020.105019

Mera, A. Y., Tabares-Gonzalez, E., Montoya-Gonzalez, S., Muñoz-Rodriguez, D. I., \& Monsalve-Vélez, F. (2020). Recomendaciones prácticas para evitar el desacondicionamiento físico durante el confinamiento por pandemia asociada a COVID-19. Universidad y Salud, 22(2), 166-177. https://doi.org/10.22267/rus.202202.188

Ministerio Educación C. y D. (MECD). (2020). Anuario de estadísticas deportivas 2020. Recuperado de http:// www.culturaydeporte.gob.es/dam/jcr:47414879- 4f95-4cae-80c4-e289b3fbced9/anuario-de-estadisticasdeportivas-2020.pdf

Ministerio de la Presidencia, Relaciones con las Cortes y Memoria Democrática. (2020a). Real Decreto 463/2020, de 14 de marzo, por el que se declara el estado de alarma para la gestión de la situación de crisis sanitaria ocasionada por el COVID-19, BOE, No 67 de 14 de marzo 25390 (2020). Recuperado de https://www.boe.es/diario_boe/ txt.php?id=BOE-A-2020-3692

Ministerio de la Presidencia, Relaciones con las Cortes y Memoria Democrática. (2020b). Real Decreto 555/2020, de 5 de junio, por el que se prorroga el estado de alarma declarado por el Real Decreto 463/2020, de 14 de marzo, por el que se declara el estado de alarma para la gestión de la situación de crisis sanitaria ocasionada por el COVID-19. BOE, no 159, de 6 de junio de 2020. Recuperado de https: / / www.boe.es/ eli/es/rd/2020/06/05/555

Ministerio de la Presidencia, Relaciones con las Cortes y Memoria Democrática. (2020c). Real Decreto 926/2020, de 25 de octubre, por el que se declara el estado de alarma para contener la propagación de infecciones causadas por el SARSCoV-2. BOE, $\mathrm{n}^{\circ} 282$, de 25 de octubre de 2020. Recuperado de https://www.boe.es/boe/dias/2020/10/25/ pdfs/BOE-A-2020-12898.pdf

Ministerio de la Presidencia, Relaciones con las Cortes y Memoria Democrática. (2020d). Real Decreto 956/2020, de 3 de noviembre, por el que se prorroga el estado de alarma declarado por el Real Decreto 926/2020, de 25 de octubre, por el que se declara el estado de alarma para contener la propagación de infecciones causadas por el SARS-CoV-2. BOE, $\mathrm{n}^{\circ} 291$, de 4 de noviembre de 2020. Recuperado de https:// www.boe.es/boe/dias/2020/11/04/pdfs/BOE-A2020-13494.pdf

Ministerio de Sanidad (2020a). Orden SND/380/2020, de 30 de abril, sobre las condiciones en las que se puede realizar actividad física no profesional al aire libre durante la situación de crisis sanitaria ocasionada por el COVID-19, BOE, $\mathrm{n}^{\circ} 121$, de 1 de mayo de 2020. Recuperado de https:// www.boe.es/eli/es/o/2020/04/30/snd380

Ministerio de Sanidad (2020b). Enfermedad por el coronavirus (COVID-19). Actualización n ${ }^{a}$ 265. Recuperado de https:/ /www.mscbs.gob.es/profesionales/saludPublica/ ccayes / alertas Actual / n Cov/documentos / Actualizacion_265_COVID-19.pdf

Moratalla, A., \& Íbero, A. (2020). El deporte ante el COVID-19: el mayor desafío de una industria casi perfecta | IDEAS LLYC. Llyc. Explorar. Inspirar. Recuperado de https:// ideas.llorenteycuenca.com/2020/05/el-deporte-anteel-covid-19-el-mayor-desafio-de-una-industria-casi-perfecta/

Moreno, M. A. (2020). Una reconversión digital para los gimnasios ante el coronavirus. La Vanguardia. Deportes. Recuperado de https: / /www.lavanguardia.com/deportes/ 20200417/48569692286/una-reconversion-digitalpara-los-gimnasios-ante-el-coronavirus.html 
Muñiz, J. (2020). El sector del fitness prevé pérdidas superiores a los 1.100 millones por el coronavirus | Deporte y Negocio. Expansión, Deporte y Negocio, Marketing Deportivo. Recuperado de https://www.expansion.com/directivos / d e porte-negocio/2020/06/17/ 5eea0c57468aeb722f8b4612.html

Mutz, M. (2021) Forced adaptations of sporting behaviours during the Covid-19 pandemic and their effects on subjective well-being. European Societies, 23(sup1), S184S198. https://doi.org/10.1080/ 14616696.2020 .1821077

OECD. (13 de mayo 2020). Respuestas políticas de las ciudades al COVID-19. Recuperado de https://oecd.org/ coronavirus/policy-responses/respuestas-politicas-delas-ciudades-al-covid-19-12646989

OMS. (2010). Recomendaciones mundiales sobre actividad física para la salud. Recuperado de https: / /apps.who.int/iris/ bitstream/hand le/10665/44441/ 9789243599977_spa.pdf?ua=1

OMS. (2020). COVID-19: cronología de la actuación de la OMS. Centro de Prensa / Detalle. Recuperado de https:/ / www.who.int/es/news-room/detail/27-04-2020who-timeline-covid-19

Owen, N., Healy, G. N., Matthews, C. E., \& Dunstan, D. W. (2010). Too much sitting: the population health science of sedentary behavior. Exercise and Sport Sciences Reviews, 38(3), 105-113. https://doi.org/10.1097/ JES.0b013e3181e373a2

Palco23. (2021). Gimnasios: ocho comunidades autónomas cierran los centros en la tercera ola. Recuperado de https:// www.palco23.com/entorno/gimnasios-ocho-comunidades-autonomas-cierran-los-centros-en-la-terceraola.html

Peake, J. M., Neubauer, O., Walsh, N. P., \& Simpson, R. J. (2017). Recovery of the immune system after exercise. Journal of Applied Physiology, 122(5), 1077-1087. https: / / doi.org/10.1152/japplphysiol.00622.2016

Pérez-Rodrigo, C., Gianzo Citores, M., Hervás Bárbara, G., Ruiz-Litago, F., Casis Sáenz, L., Arija, V.,... \& ArancetaBartrina, J. (2021). Patterns of Change in Dietary Habits and Physical Activity during Lockdown in Spain Due to the COVID-19 Pandemic. Nutrients, 13(2), 1-6. https: / / doi.org/10.3390/nu13020300

Prado, J. M., Rey, L. D., Foschi, S. A., \& Granda, D. P. (2020). Confianza de los clientes para retornar a los gimnasios. Especialización en gerencia de mercadeo. Recuperado de http: / / hdl.handle.net/10882/10276

Porras-Hilarión, M. F., Granados-Villa, M. F., \& TrujilloSastoque, J. E. (2021). Análisis de los cambios en los hábitos de consumo durante cuarentena. Recuperado de http:// repository.ean.edu.co/handle/10882/10283

Reimers, C. D., Knapp, G., \& Reimers, A. K. (2012). Does physical activity increase life expectancy? A review of the literature. Journal of Aging Research, 2012, 1-10. https: / / doi.org/10.1155/2012/243958
Rutten, G. M., Savelberg, H. H., Biddle, S. J. H., \& Kremers, S. P. J. (2013). Interrupting long periods of sitting: good STUFF. International Journal of Behavioral Nutrition and Physical Activity, 10(1), 1-3. https://doi.org/10.1186/ 1479-5868-10-1

Saris, W., Blair, S. N., van Baak, M., Eaton, S., Davies, P., Pietro, L., Fogelholm, M., Rissanen, A., Schoeller, D., Swinburn, B., Tremblay,A., Westerterp, K., \&Wyatt, H. (2003). How much physical activity is enough to prevent unhealthy weight gain? Outcome of the IASO 1st Stock Conference and consensus statement. Obesity Reviews, 4(2), 101-114. https://doi.org/10.1046/j.1467-789X.2003.00101.x

Serrano, V. (2020). Muévete en casa, entrena... pero hagámoslo bien. Revista Española de Educación Física y Deportes, 428, 15-17. Recuperado de https://www.reefd.es/ index.php/reefd/article/view/878/731

Skjong, R., \&Wentworth, B. H. (2000). Expert Judgement and Risk Perception. Recuperado de http: / / research.dnv.com/ skj/Papers/SkjWen.pdf

SGEE-Subdirección General de Estadística y Estudios. (2015). Encuesta de hábitos deportivos en España, 2015. Recuperado de http://www.culturaydeporte.gob.es/dam/ jcr:398d6e55-7bc5-4b8e-b0f3-95dd49c4997f/ Encuesta_de_Habitos_Deportivos_2015.pdf

TVE. (2020). Muévete en casa - Programa 70 (Cuerpo entero) RTVE.es. Recuperado de https: / / www.rtve.es/alacarta/ videos/muevete-en-casa/

Unesco. (2021). La UNESCO hace un llamamiento a la inversión en educación física de calidad para apoyar la recuperación postCOVID-19. Recuperado de https://es.unesco.org/ news/unesco-hace-llamamiento-inversion-educacionfisica-calidad-apoyar-recuperacion-post-covid-19

Urban Sports Club. (2020). ¿Cuáles son los deportes que más se practican durante el confinamiento? ¡Hay sorpresas!. Mujerhoy.com. Mujerhoy. Recuperado de https:// www.mujerhoy.com/vivir/fitness/202004/23/cualesson-los-deportes-mas-se-practican-en-casa-confinamiento-coronavirus-20200423141451.html

Valcarce, M. (2020). Influencia del uso de la tecnología en la adherencia de la práctica física sobre los usuarios de centros de fitness [Tesis de doctorado, Universidad de Lleida]. http: / /www.doctorat.udl.cat/en/noticies/Tesi-Influenciadel-uso-de-la-tecnologia-en-la-adherencia-de-la-practica-fisica-sobre-los-usuarios-de-centros-de-fitness /

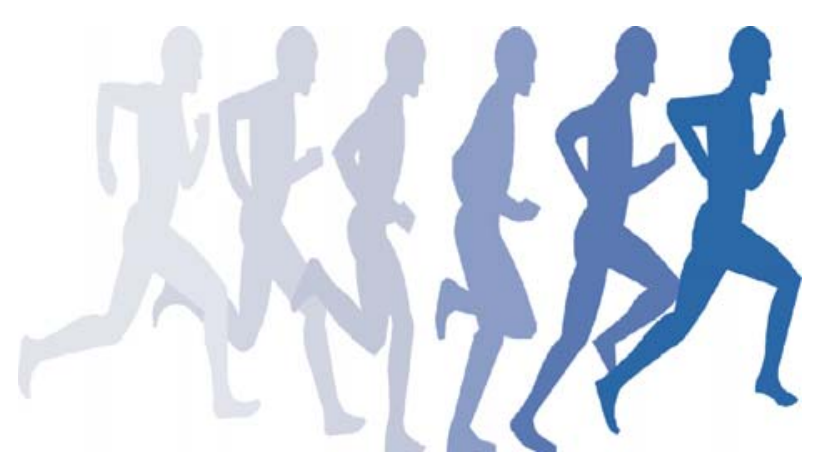

